\title{
Evolution of the retinal black sunburst in sickling haemoglobinopathies
}

\author{
GEORGE ASDOURIAN, KRISHAN C. NAGPAL, MICHAEL GOLDBAUM, \\ DIMITRIOS PATRIANAKOS, MORTON F. GOLDBERG, AND MAURICE RABB \\ From the Sickle Cell Eye Clinic of the University of Illinois Eye and Ear Infirmary, Chicago, Illinois
}

Black sunbursts are circular black chorio-retinal scars with stellate or spiculate borders, commonly found in the peripheral fundi of patients with sickle haemoglobinopathies. They are possibly found less frequently in patients with SC and sickle thalassaemia (Sthal) haemoglobinopathies (Welch and Goldberg, 1966), although in an unselected series the reported incidence of black sunbursts and similar pigmented lesions was 32 per cent of SS patients (Condon and Serjeant, 1972a), 20 per cent of Sthal patients (Condon and Serjeant, 1972b), and $4 \mathrm{I}$ per cent of SC patients (Condon and Serjeant, 1972c). Although haemorrhages and choroidal vascular occlusions have been considered as causes of these lesions (Okun, 1969; Wise, Dollery, and Henkind, $197 \mathrm{I}$; Condon and Serjeant, 1972 a, b, c; Romayananda, Goldberg, and Green, I973; Cogan, 1974) their pathogenesis has not been well documented.

This report documents the evolution of these lesions in three patients who were studied prospectively during a period of 3 years.

\section{Methods}

During the last 3 years the Sickle Cell Eye Clinic of the University of Illinois Eye and Ear Infirmary screened some 400 patients with different sickling haemoglobinopathies. Of these patients, 38 who revealed the earliest stages of sickling retinopathy (stage I, peripheral arteriolar occlusions; and stage II, peripheral arteriovenous anastomoses) (Goldberg, 1971) were included in a longitudinal study to evaluate the natural course and evolution of lesions characteristic of sickling retinopathies. Studies of these patients included a general ocular examination, indirect ophthalmoscopy, detailed fundus drawings, colour fundus photography, and fluorescein angiography of $360^{\circ}$ of the equatorial and peripheral fundus. Ophthalmoscopy, photography, and fluorescein angiography were repeated every 3 to 4 mth.

Address for reprints: Morton F. Goldberg, MD, University of Illinois, Eye and Ear Infirmary, 1855 W. Taylor Street, Chicago, Illinois, 60612, USA

\section{Results}

During the period of observation three patients developed black sunburst lesions in retinal areas that had been documented by photography as normal at the start of the observation period.

\section{CASE REPORTS}

\section{Case I}

A 19-year-old black man with sickle cell haemoglobin $\mathrm{C}$ disease, was initially screened at the Sickle Cell Eye Clinic on 3 October 1972. Haemoglobin electrophoresis revealed haemoglobin $S$ of $54 \cdot 2$ per cent and haemoglobin $\mathrm{C}$ of $45^{\circ} 8$ per cent. The haemoglobin level was $13.9 \mathrm{~g} / 100 \mathrm{ml}$, and the haematocrit reading 40.4 per cent.

The ocular history was unremarkable and corrected visual acuity was $20 / 25$ in each eye. The conjunctiva did not reveal the sickling conjunctival sign (Paton, 1962). Ophthalmoscopy revealed a variety of nonproliferative retinal lesions including retinal haemorrhages (salmon patches), chorio-retinal scars (black sunbursts), and schisis-like cavities containing iridescent spots. Both ophthalmoscopy and fluorescein angiography demonstrated extensive areas of stage I (arteriolar occlusions) and II (arterio-venous anastomoses) lesions (Goldberg, 197I) involving the majority of the equatorial retina in each eye. The patient was recruited into the longitudinal study and he has been examined every 3 $\mathrm{mth}$ with indirect ophthalmoscopy, colour fundus photography, and fluorescein angiography. On his initial examination in October 1972, the 12 o'clock meridian of the right eye did not reveal any abnormalities (Fig. I a). In November 1973, he had multiple preretinal and intraretinal haemorrhages at different stages of resorption. One of these lesions at the 12 o'clock meridian revealed gradual resorption of the haemorrhage (Fig. $1 b$ ). When he was seen in July 1974 , early pigmentary changes were evident (Fig. Ic). Two areas in the left retina at the $I$ and 4 o'clock meridians had initially revealed no abnormalities. In January I973 intraretinal haemorrhages were observed in these areas (Figs 2a, 3a). The haemorrhages were large enough to dissect into the overlying vitreous. After 6 mth these areas showed absorption of the haemorrhages with the progressive development of pigmentary changes (Figs $2 b, 3 b$ ). 

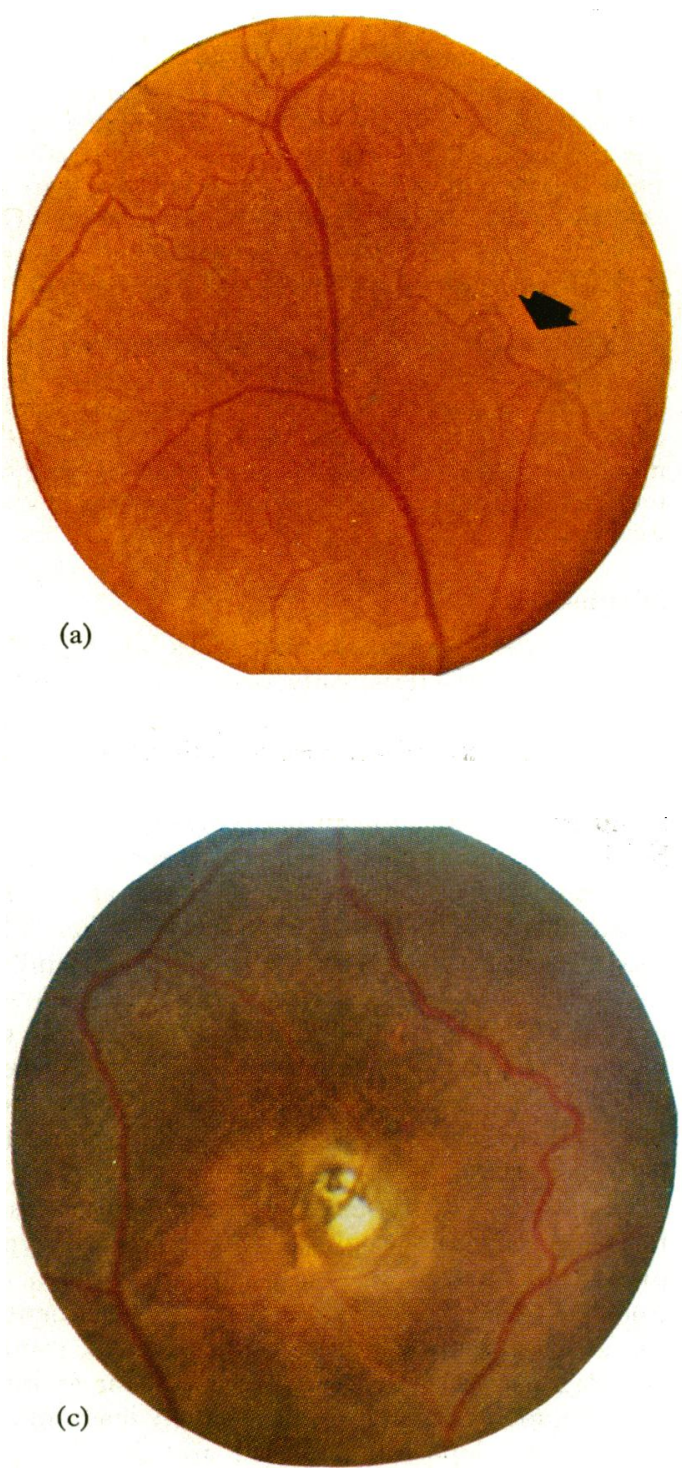

\section{Case 2}

A 27-year-old black man with sickle cell thalassaemia, was screened at the Sickle Cell Eye Clinic. He had no ocular symptoms; haemoglobin electrophoresis revealed haemoglobin $S$ of 72.8 per cent, $A_{2}$ of 5.2 per cent, and $\mathrm{F}$ of 22 per cent. The haemoglobin level was $15 \mathrm{~g} / \mathrm{r} 00 \mathrm{ml}$ and the haematocrit reading was 43 per cent. The visual acuity was $20 / 15$ in both eyes. The conjunctiva did not reveal the conjunctival sickling sign (Paton, 1962). Ophthalmoscopy revealed stage I and stage II retinopathy (Goldberg, 1971) in the right eye. Ophthalmoscopy of the left eye revealed stage I, II, and III (sea-fan neovascularization) retinopathy (Goldberg, 1971). Fluorescein angiography of the right eye confirmed the

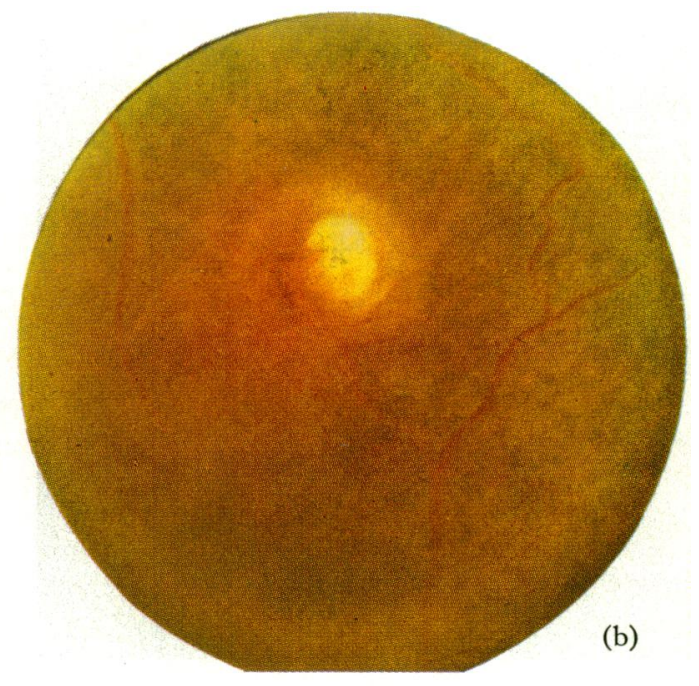

FIG. I Case I, right eye. (a) Normal-appearing 12 o'clock meridian in October 1972. Arrow indicates site of future intraretinal haematoma.

(b) Same area after I yr with evidence of resorbing intraretinal haemorrhage. Haemorrhage has also broken into vitreous, causing slight haze. (c) Same area in $\mathfrak{Y}$ uly 1974. Pigmentary changes have appeared in centre of lesion with evidence of schisis cavity around it

presence of arteriolar occlusions and arterio-venous anastomoses. Fluorescein angiography of the left eye revealed occluded arterioles and arterio-venous anastomoses as well as perfusion and leakage of dye from the sea fan. On follow-up examination, the i i o'clock area of the left eye, which had been initially normal, showed an intraretinal haemorrhage, which ro mth later started developing pigmentation characteristic of black sunbursts.

\section{Case 3}

A 23-year-old black man with sickle cell haemoglobin C disease, was seen at the Sickle Cell Eye Clinic on 5 December 1972, with a history of blurred vision in 


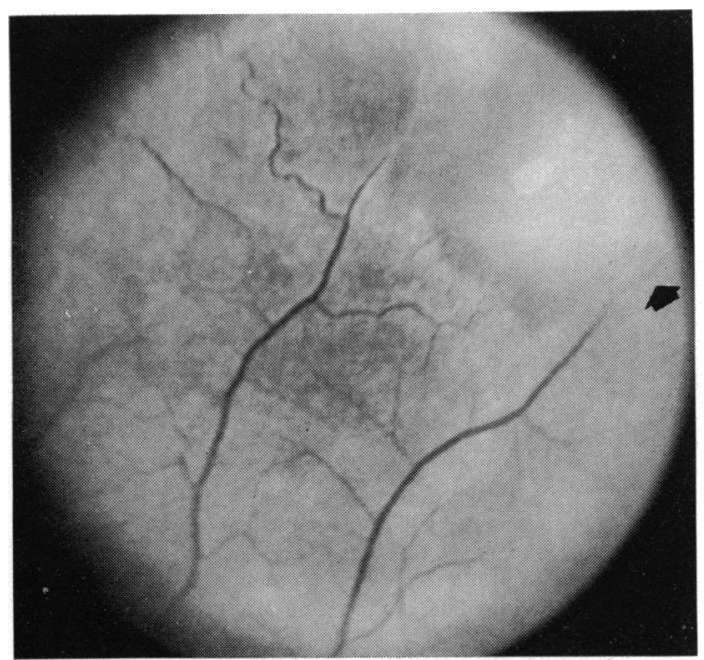

(2a)

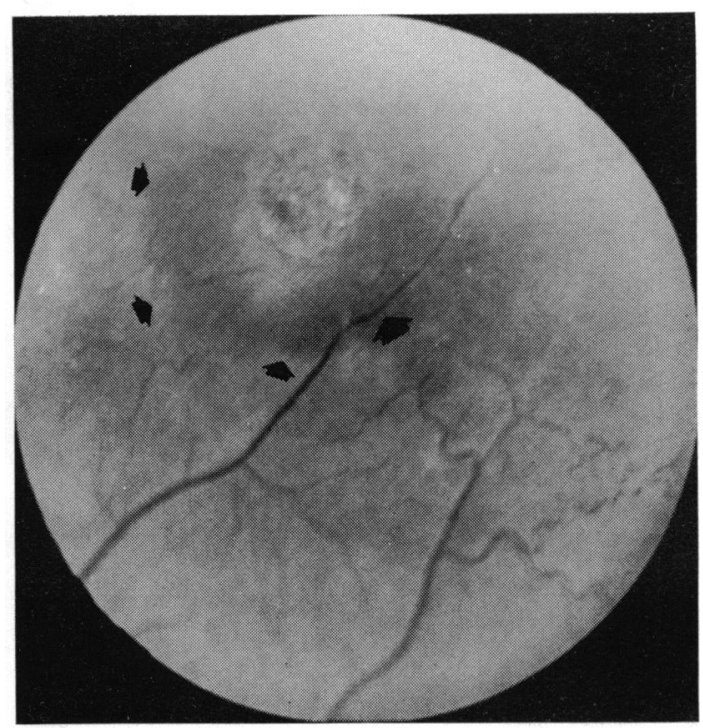

(2b)

FIG. 2 Case I, left eye. (a) I o'clock meridian in January 1973 revealing intraretinal haemorrhage with overlying vitreous haze. Arrow indicates reference vessel (see Fig. 2b). (b) Same area after 6 mth. Chorio-retinal scar with iridescent spots has resulted. Smaller arrows indicate boundaries of schisis cavity caused by resorption of haematoma. Larger arrow indicates reference vessel (see Fig. 2a)

the right eye. Haemoglobin electrophoresis revealed haemoglobin $S$ of 53.1 per cent and haemoglobin $C$ of $46 \cdot 9$ per cent.

Corrected visual acuity was $20 / 30$ in the right eye and $20 / 25$ in the left eye. There was no conjunctival sickling sign. Ophthalmoscopy revealed stage I, II, and III retinopathy in both eyes and evidence of an old vitreous haemorrhage in the right eye. The patient was subsequently admitted to hospital several times and the neovascularization was treated with cryotherapy.

Of interest was an area at the 2 o'clock meridian in the right eye. At the initial examination this area appeared to be normal (Fig. $4 a$ ). One year later there was evidence of a previous intraretinal haemorrhage (Fig. $4 b)$. Two years after the initial examination this area revealed the start of pigmentation characteristic of a black sunburst (Fig. $4 c$ ).

Another area of interest in this patient was the 9 o'clock meridian of the same eye. In February 1973, an intraretinal haemorrhage (salmon patch) was evident adjacent to a well-developed, small black sunburst. One mth later there was gradual absorption of the haemorrhage and $7 \mathrm{mth}$ later minimal pigmentary changes were evident.

\section{Discussion}

Black sunbursts are circular black chorio-retinal scars that characteristically occur in the fundi of patients who have various types of sickle cell anaemia. These well-developed lesions have typical features distinguishing them from somewhat similar-appearing inflammatory lesions; the sunbursts are usually round or ovoid, ranging in size between 0.5 and 2 disc diameters, and are characteristically located in the equatorial fundus. They usually have stellate or spiculate borders and are frequently associated with refractile yellowish granules. Their configurations range from tiny pigmented spots to large multi-spiculated scars (Fig. $5 a-c$ ). Because of their characteristic peripheral location these lesions usually do not interfere with vision.

Histologically, these lesions represent areas of focal retinal pigment epithelial hypertrophy, hyperplasia, and migration. Occasionally there is a distinct perivascular localization of the pigment in the sensory retina. The sensory retina is thinned and degenerated with loss of its lamellar architecture. Pigmented, haemosiderin-laden macrophages and diffuse deposition of iron are also found in these lesions (Romayananda and others, 1973).

The pathogenesis of these lesions has remained speculative. Retinal arteriolar occlusions had previously been incriminated because of the presence of arterioles which apparently supplied the area of the retina involved by the sunbursts (Welch and Goldberg, I966); however, arteriolar feeders could not be found in all cases. A blow-out of preretinal, intraretinal, or subretinal haemorrhage, with subsequent reparative processes in the retina, had also been proposed as the cause of the black sunburst lesions (Okun, 1969; Condon and Serjeant, 1972b; Romayananda and others, 1973).

Okun (1969) reported a case where an initial salmon-coloured preretinal haemorrhage, which was accompanied by adjacent subretinal bleeding, 

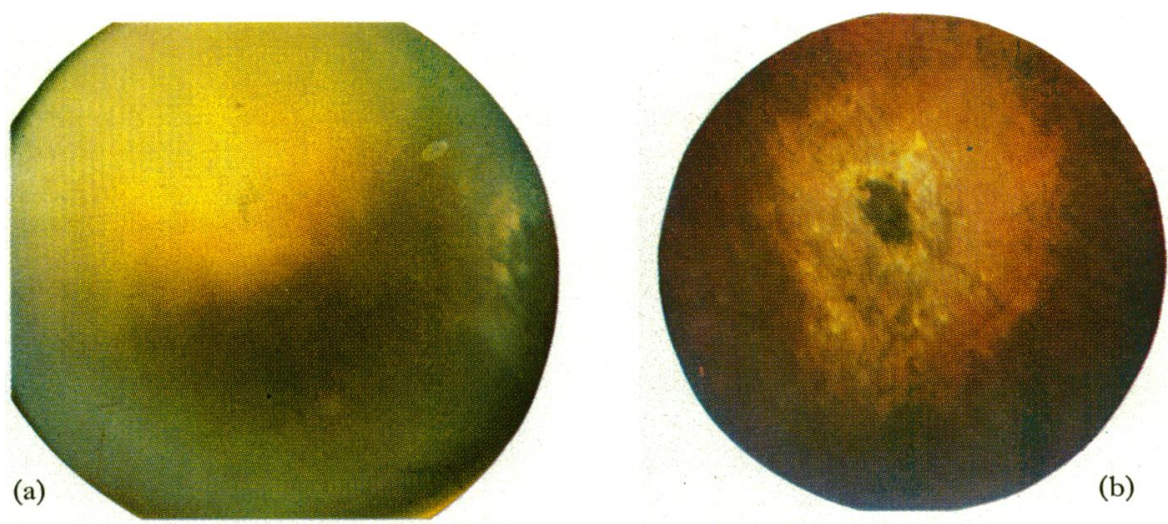

FIG. 3 Case I, left eye. (a) 4 o'clock meridian in fanuary 1973 revealing large intraretinal haemorrhage which has dissected into vitreous. (b) Same area after 6 mth. Characteristic sunburst has resulted
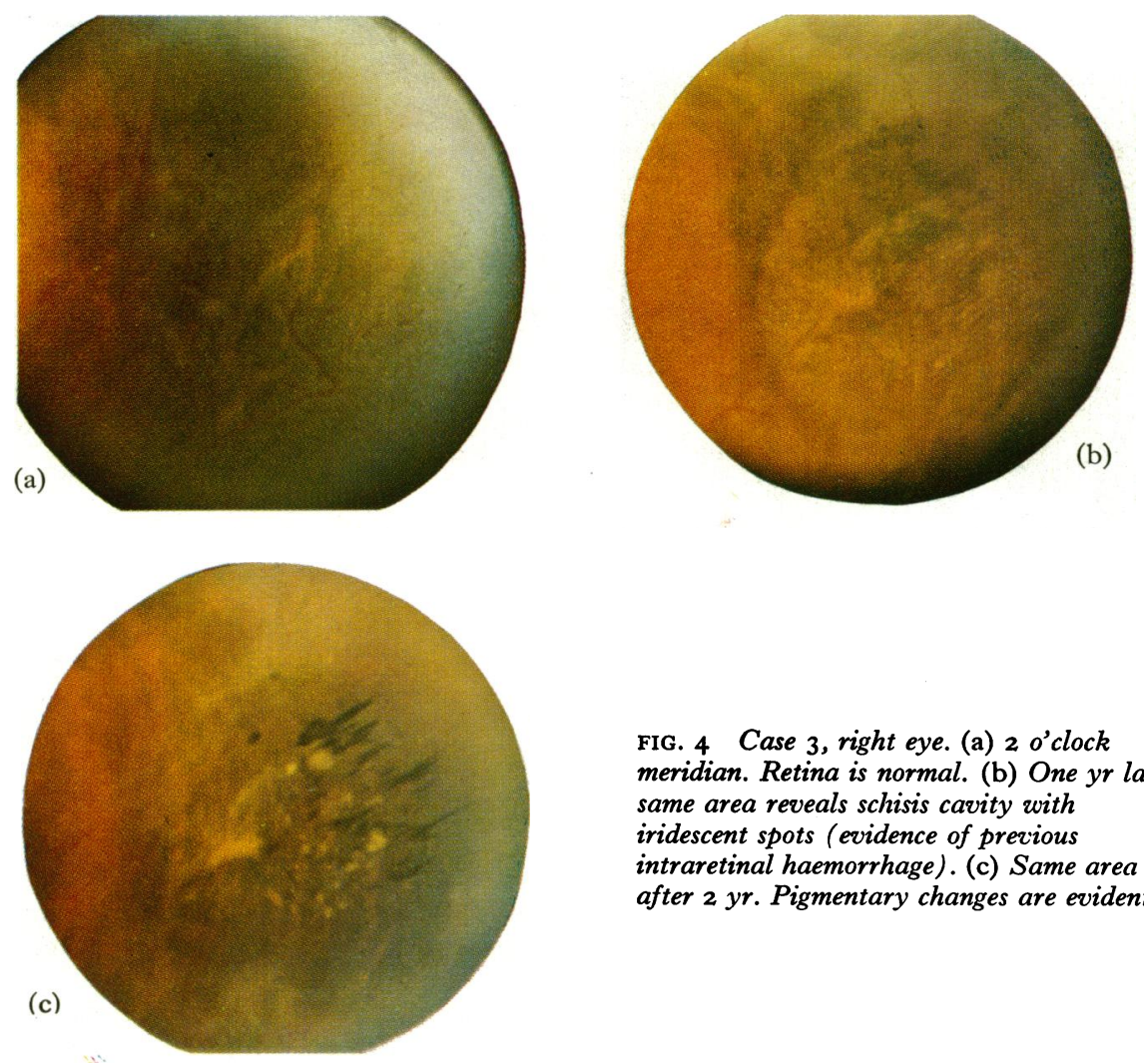

FIG. 4 Case 3, right eye. (a) 2 o'clock meridian. Retina is normal. (b) One yr later same area reveals schisis cavity with iridescent spots (evidence of previous intraretinal haemorrhage). (c) Same area after 2 yr. Pigmentary changes are evident

subsequently disappeared to leave a brownish area with iridescent spots. This was called a chorioretinal scar, but there was no evidence of excessive pigment and no further follow-up examinations of the area were made. 
The intraretinal haemorrhage was large enough to dissect into the vitreous in some cases, and it also apparently dissected through the photoreceptors into the potential space between the sensory retina and the pigment epithelium (the subretinal space). Evidence of retinal haemorrhage dissecting into the subretinal space has also been documented in angiomatosis retinae (Goldberg and Duke, r968). The presence of blood adjacent to the retinal pigment epithelium presumably stimulates it to proliferate and migrate. With the absorption of the small haematoma within the sensory retina, schisis cavities develop. Iridescent spots, which

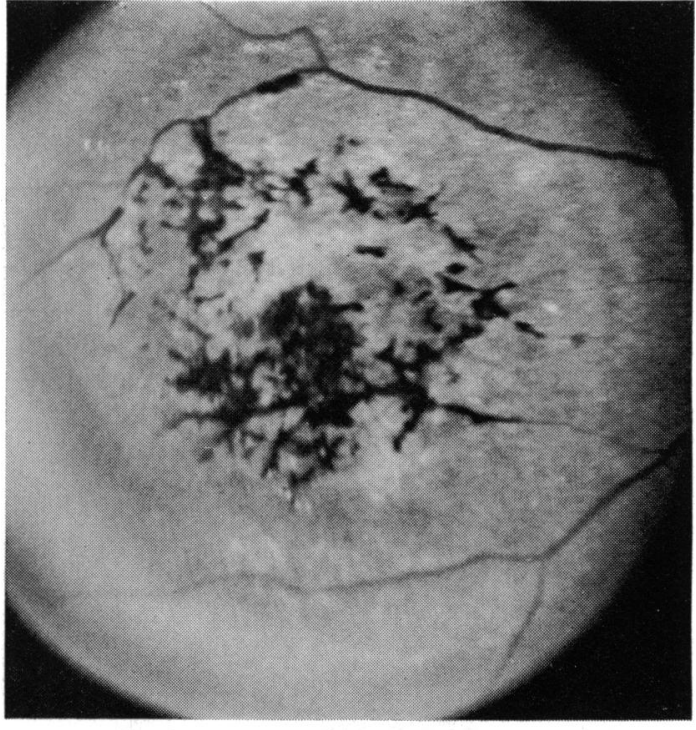

(5c)

FIG. 5 (a-c) Different configurations of well-developed black sunbursts

represent iron-laden macrophages, are often found within these cavities. The cavities surrounding the sunburst remain as evidence of the initiating intraretinal haematoma.

The absence of the retinal vasculature surrounding certain black sunbursts is readily explained by the known repetition in sickling patients of arteriolar occlusions and proximal propagation of intravascular clots with drop-out of surrounding capillaries. Some sunbursts are therefore left in areas of avascular retina with no apparent connexions to the adjacent retinal vasculature.

Certain intraretinal haemorrhages, on the other 


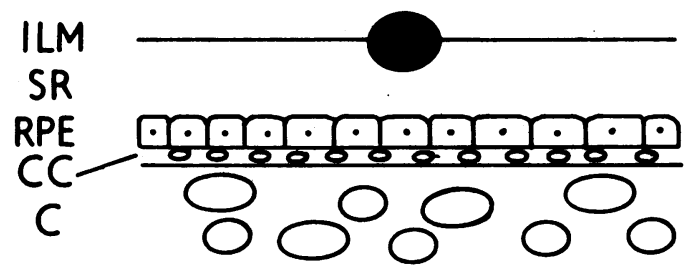

Salmon patch hoemorrhage

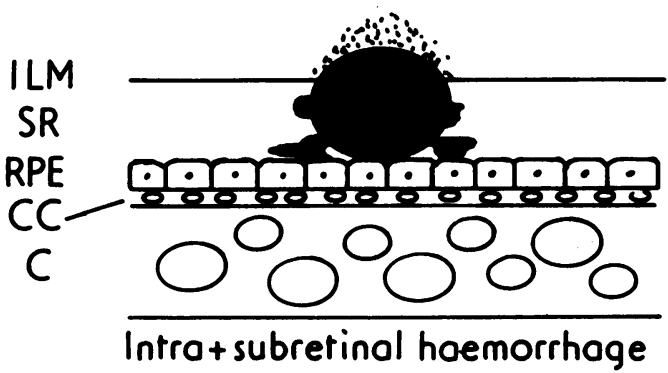

$\underline{A}$

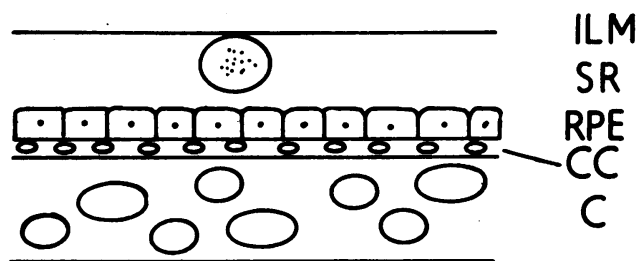

Schisis covity with iridescent spots
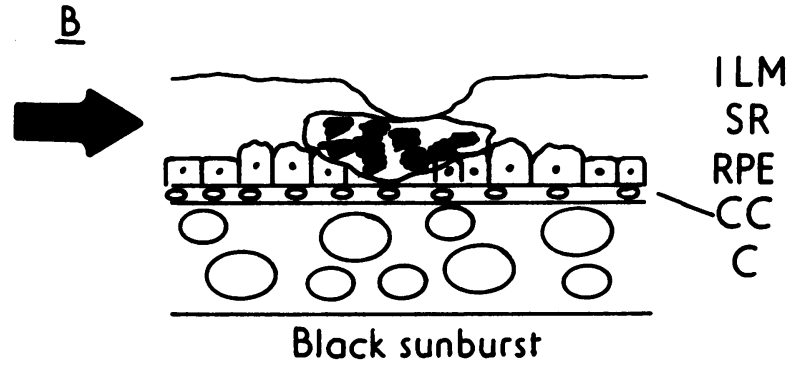

FIG. 6 Schematic interpretation showing a salmon patch haemorrhage becoming schisis cavity and (b) large intraretinal haemorrhage dissecting into subretinal space and turning into black sunburst. ILM, internal limiting membrane; SR, sensory retina; $R P E$, retinal pigment epithelium; $C C$, choriocapillaris ; $C$, choroid

hand, do not dissect into the subretinal space. Their resorption leaves behind schisis cavities with iridescent spots, but without evidence of pigmentary disturbance (Fig. 6).

The causative role of retinal arteriolar occlusions has been disputed by some authors with the argument that branch and central retinal artery occlusions are never associated with pigment alterations (Wise and others, 1971; Cogan, 1974). However, in these circumstances, the retinal pigment epithelium is not traumatized and therefore does not respond by proliferation or migration. Interference with the choriocapillaris circulation has been suggested as the cause of black sunbursts (Wise and others, 1971; Cogan, 1974), but evidence for this hypothesis is lacking. Fluorescein angiography is usually of no great diagnostic help during the acute stage, when the haemorrhage obscures the involved vessels. In healed lesions fluorescein angiography reveals areas of hyper- and hypofuorescence depending on the amount of pigment clumping and pigment dispersion (Fig. 7). Adjacent capillary bed drop-out is usually present, and is evidence of previous retinal vasculature occlusion. In many cases the major arteriole responsible for the initial blow-out haemorrhage can also be seen.

Acute occlusions of choroidal capillaries do occur in other cases and have been noteworthy in patients with severe systemic hypertension. These occlusive phenomena result in pigmented areas in the fundus known as Elschnig's spots (Klien, 1968; Morse, 1968; Burian, 1969), which differ from the black sunburst lesions, clinically as well as pathologically. Clinically, the typical, healed Elschnig spot is an isolated, round, yellowish area, one-sixth to one-third the papillary diameter with a pigmented centre. It is most commonly seen in the vicinity of the optic disc or the retinal midperiphery. Black sunbursts are usually larger and are generally located at the retinal equator. A characteristic sunburst in the posterior pole has

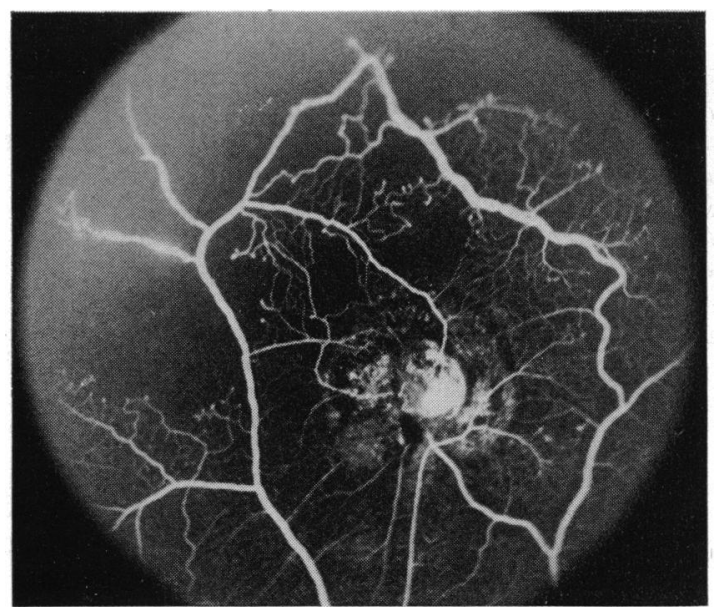

FIG. 7 Case I, Fluorescein angiogram of black sunburst (cf. Fig. I c). Pigmentary changes evident as hypo- or hyperfluorescent areas. Capillary abnormalities evident. No neovascular tissue or leakage of dye 
yet to be seen. Pathologically, the differences between these lesions are even more marked. An Elschnig spot represents a clump of pigment; the surrounding depigmented epithelial cells constitute the yellow-orange halo. The choriocapillaris shows foci of fibrosis, obliteration, and residual ghost capillary outlines. The overlying sensory retina is usually normal. In contrast, the yellowish halo around the black sunburst represents the boundaries of a schisis cavity that often contains iron-laden macrophages; the choriocapillaris does not show any occlusive phenomena and the retinal architecture is distorted because of the previous intraretinal haemorrhage. The black sunburst also differs in several respects from the extensive chorio-retinal degenerations that have been reported to be the sequelae of ciliary and choroidal artery occlusions (Condon, Serjeant, and Ikeda, 1973), in that the latter lesions occupy large sectoral areas of the fundus. They are also characterized by advanced choroidal and retinal pigment epithelial atrophy with a grey-green background, through which choroidal vessels are usually visible.

In the differential diagnosis of the black sunburst one should also consider inflammatory disease that can cause chorio-retinal scars, such as toxoplasmosis, syphilis, and presumed histoplasmosis.
Toxoplasmic chorio-retinal scars are often found in the posterior pole as well as the retinal periphery. Apart from differences of locality, these lesions are often characterized by extensive fibrosis or by intense pigment proliferation and clumping around their periphery, leaving a central atrophic region through which the choroid and sclera are exposed. Satellite scars are sometimes present. The retinal lesions in presumed histoplasmosis are very small, punched-out, pale, slightly pigmented, discrete foci, and are usually located in the retinal periphery. Disseminated chorio-retinitis as a result of syphilis may be manifested as varying types of chorio-retinal pigmentation, irregular masses, and dispersed or confluent areas of scarring. Pigment sheathing of veins and narrowing of retinal arteries are often present (Ballantyne and Michaelson, 1973).

\section{Summary}

In a prospective study of 38 patients, who were initially selected as being at an early stage of sickling retinopathy, three developed circular black chorio-retinal scars (black sunbursts) during a period of 6 to 24 months. These lesions appear to be the sequelae of intraretinal and subretinal haemorrhage. They occur in the fundus periphery and do not interfere with vision.

\section{References}

ballantyne, A. J., and michaelson, I. c. (1973) 'Textbook of the Fundus of the Eye'. Williams \& Wilkins,

Baltimore

BURIAN, H. M. (1969) Amer. F. Ophthal., 68, 412

COGAN, D. G. (1974) 'Ophthalmic Manifestations of Systemic Vascular Disease'. Saunders, Philadelphia

CONDON, P. I., and SERJEANT, G. R. (1972a) Amer. F. Ophthal., 73, 533

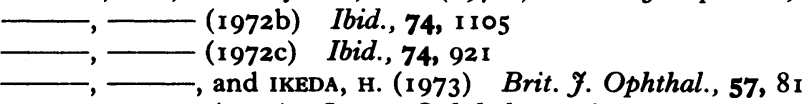

GOLDBERG, M. F. (1971) Invest. Ophthal., 12, 633 , and DUKE, J. R. (1968) Amer. F. Ophthal., 66, 693

KLIEN, B. A. (1968) Ibid., 66, 1069

MORSE, P. H. (1968) Ibid., 66, 844

OKUN, E. (1969) Docum. ophthal. (Den Haag), 26, 574

Paton, D. (1962) Arch. Ophthal., 68, 627

romayananda, N., Goldberg, M. F., and Green, w. R. (1973) Trans. Amer. Acad. Ophthal. Otolaryng., 77, 652

WELCH, R. B., and GOLDBERG, M. F. (1966) Arch. Ophthal., 75, 353

WiSE, G. N., Dollery, C. T., and Henkind, P. (1971) 'The Retinal Circulation'. Harper \& Row, New York 